Multilevel models for social networks: Hierarchical Bayesian approaches to exponential random graph modeling

Andrew J. Slaughter

US Army Research Institute for the Behavioral and Social Sciences ${ }^{1}$

Laura M. Koehly

National Human Genome Research Institute ${ }^{2}$

Author Note

The views, opinions, and/or findings contained in this article are solely those of the authors and should not be construed as an official Department of the Army or DOD position, policy, or decision, unless so designated by other documentation. Exemplar data used in to illustrate methods presented in this paper were obtained through support from the Breast Cancer Research Program, The University of Texas M.D. Anderson Cancer Center. The completion of this article was partially supported by the Intramural Research Program of the National Human Genome Research Institute at the National Institutes of Health.

\footnotetext{
${ }^{1}$ Corresponding author. Mailing address: 6010 Frankford St., \#221, Aberdeen Proving Ground, MD 21005, USA. Tel: 443-395-8127. Email: andrew.j.slaughter.civ@mail.mil

${ }^{2}$ Mailing address: Building 31, Room B1B54, 31 Center Dr, MSC 2073, Bethesda, MD 20892 , USA. Email: koehlyl@mail.nih.gov
} 


\section{Multilevel models for social networks: Hierarchical Bayesian approaches to exponential random graph modeling}

\section{Introduction}

One important goal of network analysis is the ability to statistically model ties in social or other networks. Typically, this is done by making inferences about potential types of dependencies, characterized by particular structural features or patterns within the network. These patterns can be used to help understand the various processes - such as social or cognitive processes - that networks exhibit in particular contexts. Although researchers are typically interested in understanding general processes across networks and contexts, network research has often focused on the analysis of individual networks.

There are a number of possible reasons for this tendency. One is the relative difficulty in collecting data from multiple networks - especially networks large enough to display more than the very simplest types of structure. Another is the fact that much of network analysis has historical roots in areas like sociology and anthropology - disciplines with prominent histories of research involving indepth qualitative and quantitative studies of specific groups and social contexts. Thus, these studies, while aimed at understanding general social phenomena, tend to focus on individual network systems, rather than multiple networks. The third - and perhaps primary reason - is the (relative) lack of statistical tools to appropriately handle data from multiple networks of varying sizes.

The focus on analyzing individual networks is not at all unreasonable. However, it is becoming progressively easier to collect network data from large numbers of groups. Similarly, network methods have continued to proliferate in disciplines where scientific methods are not grounded in making general inferences based on the individual analysis of a small number of ad-hoc networks. Rather, such disciplines aim to understand systematic structural patterns observed across many groups, and differences observed between groups. There have been a number of advances in methods for the analysis of collections of networks (Lubbers \& Snijders, 2007; Wang, Robins, Pattison, \& Lazega, 2013; Zijlstra, van Duijn, \& Snijders, 2006); in their current form, these methods are useful in a number of ways, and even quite powerful, but have limitations in how they can be used to model variation in relational tendencies within and across groups. 
The purpose of the current paper is to present some new options for researchers interested in studying networks in a multilevel context, based on extensions to the usual exponential random graph model (ERGM) using a hierarchical Bayesian approach. We first describe models for the special case in which multiple independent sets of networks have been observed. Then, options that account for increased complexity illustrated by true "multilevel" networks (e.g., networks between nodes at different levels of analysis) are presented. Several empirical examples are then presented, and potential applications and future extensions are discussed.

\section{Extending the ERGM}

There are a wide variety of methods and metrics available to researchers who are interested in exploring the structural properties of networks. However, for those interested in making inferences about such properties, stochastic approaches, which provide a basis for statistical tests, are required. Perhaps the most widely used approach is the ERGM.

The ERGM is a conceptually powerful modeling framework for the analysis of network data. Its development, rationale, and use has been covered extensively elsewhere (for example, see Lusher, Koskinen, and Robins 2013 for a general overview), but briefly, ERGMs allow researchers to specify sufficient statistics in the form of counts of so-called "graph motifs." These motifs are typically defined based on the researchers' beliefs about the potential nature and sources of dependencies between and across particular sets of actors and their dyadic relations.

The basic ERGM may be written in terms of the following equation:

$$
P(G=g \mid \theta)=\frac{e^{\theta z(G)}}{k(\theta)}
$$

This equation defines the probability of observing any particular graph realization as a function of a vector of sufficient statistics defined on the observed graph, $z(g)$, a vector of ERGM parameters, $\theta$, and an intractable normalizing constant, $k(\theta)$. For notational convenience, we define the distribution of a set of network ties given by the above ERGM using the following:

$$
g \sim F(\theta, z)
$$

ERGMs are tremendously flexible models, which are easily extended to much more complicated structures than simple graphs. Extensions include models for networks with multiple types of ties, various types of hypergraphs (which include two-mode networks and cognitive social structures as special cases), networks with temporal dependencies, and multilevel "networks of networks" (c.f., Desmarais \& Cranmer, 2012; Koehly \& Pattison, 2005; Wang et al., 2013; Wang, Sharpe, Robins, \& Pattison, 2009). 
Such models are extremely powerful, but with a handful of notable exceptions, they have generally been oriented towards the study of single networks. They have not been designed to model the variability in structure across collections of (possibly independent) networks. However, for researchers in many fields, the ability to model between-group variability may be of particular interest. For example, in the area of team and small-group research, researchers may be especially interested in understanding how group-level contextual factors such as training and leadership relate to variability in relational tendencies at the group level. In the area of public health, where intervention efforts might be focused on activating interpersonal relationships within a group, approaches that can compare intervention and control groups with respect to interpersonal interactions, or network structure, are necessary.

Traditionally, extensions to ERGMs have been difficult to develop and apply, due in large part to the presence of an intractable normalizing constant in the likelihood function. However, recent work in Markov Chain Monte Carlo (MCMC) estimation now offers a variety of tools to deal with the issue. These include algorithms for approximate Bayesian computation (ABC; Marin, Pudlo, Robert, \& Ryder, 2012) and MCMC approaches based on auxiliary variables (e.g., Caimo \& Friel, 2011; Jin, Yuan, \& Liang, 2013; Liang, 2010).

These Bayesian techniques provide an elegant way of handling estimation for statistical models like ERGMs. Perhaps more importantly, these algorithms fit naturally into the hierarchical Bayesian paradigm and applications to multilevel models. In so doing, these algorithms afford an opportunity to more easily develop new types of multilevel models that take advantage of the utility of the ERGM framework, while offering additional flexibility for exploring variability in structure across networks and levels. Several simple examples are described in the next sections.

\subsection{Modeling independent samples of networks}

When faced with the analysis of multiple independent networks, two primary approaches have been used. One approach is the random effects p2 model (Zijlstra et al., 2006). Although this model allows for the estimation of a single comprehensive "random effects" model for networks via IGLS (iterative generalized least squares) or MCMC, the underlying p2 model is limited in the type of structures and dependencies that can be modeled, allowing for modeling the effects of density, reciprocity, as well as actor-specific effects on sending and receiving ties, and covariates which can predict the actor-specific effects.

The second, and more widely used approach, is the meta-analytic approach (see Lubbers \& Snijders, 2007, for example). The meta-analytic approach is essentially a two-stage method. That is, given some set of $n$ individual networks, the analyst first estimates $n$ different ERGMs using standard statistical techniques. For each network $i$, this results in a vector of ERGM parameter estimates $\theta_{i}$ and an estimate of the uncertainty for those estimates $\Sigma_{s e(i)}$.

Different variations on the meta-analytic approach are possible, including both fixed-effects and random-effects approaches. In the random-effects meta- 
analytic approach, we assume that there are some unobserved "true" random effects $\mu_{i}$ given by the following set of distributions:

$$
\begin{aligned}
\theta_{i} & \sim \operatorname{MVN}\left(\mu_{i}, \Sigma_{s e(i)}\right) \\
\mu_{i} & \sim \operatorname{MVN}\left(\theta_{f}, \Sigma_{r}\right)
\end{aligned}
$$

In this model, $\theta_{f}$ represents the vector of fixed effects and $\Sigma_{r}$ is the variancecovariance matrix associated with the random effects. In a Baysian context, we place appropriate priors on $\theta_{f}$ and $\Sigma_{r}$ to complete the model specification. The posterior distribution of the parameters is then given by

$$
P\left(\mu_{i}, \theta_{f}, \Sigma_{r} \mid \theta_{i}, \Sigma_{s e(i)}\right) \propto \prod_{i=1}^{n}\left\{P\left(\theta_{i} \mid \mu_{i}, \Sigma_{s e(i)}\right) P\left(\mu_{i} \mid \theta_{f}, \Sigma_{r}\right)\right\} P\left(\theta_{f}\right) P\left(\Sigma_{r}\right)
$$

Despite the extreme simplicity of the two-step approach, there is much to recommend its use. For applied researchers, it has the benefit of being conceptually straightforward, and can be easily implemented using current software packages such as as statnet (Handcock, Hunter, Butts, Goodreau, \& Morris, 2003), WinBUGS (Lunn, Thomas, Best, \& Spiegelhalter, 2000) or Stan (Stan Development Team, 2013). Moreover, estimates from such an approach are not expected to be unreasonable: the use of random effects approaches to the analysis of aggregate data have been extensively developed and widely used in many disciplines. That being said, two-step approaches do have potential drawbacks.

First, it is not immediately clear that the two-stage method based on random effects meta-analysis will, in practice, always produce identical results to simultaneous estimation across the full data set. This question (in a non-network context) has received some attention in the medical literature, where several researchers have investigated the meta-analysis (including random effects logistic models) of aggregate data (e.g., based on summary statistics) vs. the metaanalysis of original individual-level data. In general, the two methods ought to provide identical results when estimating fixed effects and variance parameters using a random effects meta-analysis based on summary statistics. This is usually the case, but differences can sometimes be observed across different types of random effects approaches (Debray, Moons, Abo-Zaid, Koffijberg, \& Riley, 2013; Dutton, 2011).

A second potential limitation, related to the first, is that the performance of the two-step method necessarily depends on the quality of the underlying group-specific parameter estimates. To the extent that parameter estimates and their estimated standard errors are inaccurate or poorly estimated, it becomes more difficult to justify the meta-analytic procedure. For example, if one group has parameter estimates that are statistically suspect or undefined (due to, e.g., peculiarities of the original estimation methods used), then we might also expect estimates of the meta-analytic parameters to be incorrect. For many commonlyused models, this is not a typical worry - group-specific parameter estimates are 
easily found, and the associated models and algorithms are often surprisingly robust to potential problems with the data.

However, not all models are so robust. A classic example of this is logistic regression, where the ability to find good parameter estimates (or sometimes any estimates whatsoever) can be hampered by a number of (often related) issues. These include sparseness in the data, collinearity in the predictors, or separation. For example, separation describes a situation where a predictor is perfectly collinear with the binary outcome - e.g., all males having one outcome and all females having a different outcome.

In such situations, maximum likelihood estimates for the parameters in question may not exist (Heinze \& Schemper, 2002), and output from standard statistical packages may be highly suspect. Dealing with separation can be difficult: researchers may fix estimates to $\pm \infty$ for purposes of computing other parameters, drop parameters entirely, or use more advanced models to provide regularization (e.g., Bayesian models for logistic regression) in an effort to produce more realistic estimates of coefficients and their standard errors. Of course, when such issues are present, it can complicate estimation of a meta-analytic model. For example, if parameter estimates do not exist for all groups.

Along similar lines, ERGMs have their own well-known issues when it comes to separation, degeneracy, and finding good estimates for parameters. Dealing with these issues is not simple, and can require a number of strategies to remedy 1 . In practice, such issues can make it difficult to successfully apply the standard two-step method; this is especially true in situations involving large numbers of networks (since we can expect that the likelihood that at least one "degenerate" solution occurs will increase with the number of networks) and may be related to network size (e.g., Hunter, Krivitsky, \& Schweinberger, 2012).

Finally, the two-stage method may not provide the optimal handling of missing data (including missing tie data). Specifically, a two-stage model must handle missingness at the level of each group-specific model; while standard techniques (such as Bayesian estimation) may be used for imputing missing ties, in a two-stage model these imputations are only able to take into account structural information at the level of the individual network, and are unable to take advantage of the full set of information available across all networks. To the extent that real data often contain missing responses, this is a potentially important limitation.

\footnotetext{
${ }^{1}$ For example, one strategy may be to make use of increasingly complex forms of structural dependence (e.g., "alternating k-star"-type structures). However, these can increase computational time significantly and potentially complicate the interpretation of results; and in any case, does not guarantee successful estimation. Another common response is to tweak various aspects of the model-fitting algorithm, such as starting values, burn-in times, thinning values, etc. Such tweaking also provides no guarantee of improved results, but does increase the time required to develop and fit models. Alternatively, Caimo and Friel (2011) discuss how MCMC approaches in a Bayesian context may potentially help avoid degenerate solutions.
} 


\subsection{A random coefficient extension to the ERGM}

As an alternative to the two-step process described above, it is also possible to develop a full hierarchical Bayesian approach to simultaneously model multiple sets of networks. This is made possible by recent developments in MCMC techniques for the Bayesian estimation of models with intractable normalizing constants (Liang, 2010; Caimo \& Friel, 2011). The application of hierarchical Bayesian models has the potential to help alleviate some of the concerns raised above.

First, it allows for improvements in the handling of missing data by using all available information across groups. Second, and most importantly, it allows for the incorporation of prior information about possible distributions of model parameters. This can include, for example, informative priors based on previous research, weakly informative priors used for purposes of regularization (e.g., Gelman, Jakulin, Pittau, \& Su, 2008), or priors that flexibly incorporate knowledge about the distribution of parameters in other groups via e.g., partial pooling.

The meta-analytic approach presented above essentially involves fitting a separate ERGM to each network, resulting in what are sometimes called "unpooled" estimates (Gelman et al., 2013). Such estimates may provide good fit to any individual network, but can tend to overstate the level of variability across groups (Gelman et al., 2013). In the context of ERGMs, it is also possible to form what might be called "completely pooled" estimates - for example, by treating the set of networks as one large network with structural zeros assumed for between-group ties. Such completely pooled estimates implicitly assume that there is a single set of ERGM parameters common to all groups, and as such do not consider potential variability across groups. An example of fitting multi-network ERGMs (both pooled and unpooled) can be found in Anderson, Wasserman, and Crouch (1999).

Hierarchical models provide for partial pooling, a half-way point between unpooled and completely pooled estimates. Specifically, hierarchical models allow for the estimation of group-specific effects that take into account data from across all groups, where the data is allowed to control the extent of pooling. This results in shrinkage estimates of the network-specific parameters, where effects in each group are "shrunk" towards a common value, with the degree of shrinkage typically dependent on the standard error of the estimates and the variance in estimates across groups. Shrinkage estimators in multilevel models (cf., Morris \& Lysy, 2012) can provide significant improvements in predictive accuracy (Steyerberg, Eijkemans, \& Habbema, 2001) and protect against overfitting.

Taking this into account, we motivate a simple random coefficient exponential random graph model (RCERGM) in the following way: given a set of networks, we assume exchangeability, where graph-level parameters are presumed to be conditionally independent, given some common underlying distribution. We assume that individual graphs $g_{i}$ are drawn from a distribution governed by the usual ERGM specification, and that the parameters of the network-specific 
ERGM $\theta_{i}$ are drawn from a common distribution; in the case of correlated random effects, a multivariate distribution with mean $\theta_{f}$ and variance-covariance matrix $\Sigma_{r}$ :

$$
\begin{aligned}
g_{i} & \sim F\left(\theta_{i}, z_{i}\right) \\
\theta_{i} & \sim \operatorname{MVN}\left(\theta_{f}, \Sigma_{r}\right)
\end{aligned}
$$

We complete the Bayesian specification with the addition of appropriate priors for $\theta_{f}$ and $\Sigma_{r}$. This represents a simple and direct application of standard multilevel modeling to ERGMs for multiple networks. Of course, the appropriateness of this model rests on the adequacy of the underlying assumptions, such as the conditions under which exchangeability can be expected to hold, or the appropriateness of the particular distribution chosen to model the random effects.

In some cases, a hierarchical model may only be exchangeable taking into account covariate information (Gelman et al., 2013). For instance, we might expect that the scale of ERGM parameters will tend to vary based on the size of the network, making an assumption of simple exchangeability inappropriate for cases involving networks with significant variability in size. Moreover, the inclusion of such covariates is often of great theoretical importance, such as the extent to which structural tendencies in teams and small groups are associated with differences in group culture or leadership, or the effects of planned interventions on interpersonal interactions within a group, such as families.

For this reason, we may wish to fit models that include network-level covariates. Ignoring the necessary priors, we can define such a model using the following set of equations:

$$
\begin{aligned}
g_{i} & \sim F\left(\theta_{i}, z_{i}\right) \\
\theta_{i} & \sim M V N\left(\mu_{i}, \Sigma_{r}\right) \\
\mu_{i} & =X_{i} \beta
\end{aligned}
$$

In these equations, $\mu_{i}$ represents the vector of predicted ERGM parameters for graph $g_{i}$, which we can define as a function of an intercept and some set of (possibly centered) covariates $X_{i}$ for network $g_{i}$, and a set of regression parameters $\beta$. When only an intercept term is included, this reduces to the basic RCERGM.

\subsection{Modeling multilevel networks}

The models discussed to this point have been geared towards studying samples of independent networks, where variation in structural tendencies might be explained by graph-level covariates and simple sampling variability. This is a common and important use case, especially in the context of team and small group research. However, it is also restrictive in certain respects. 
We might wish to relax these restrictions in several ways. For example, just as individuals in groups may be connected by complex patterns of relationships, groups themselves may be connected in complex ways. Such data structures are sometimes referred to as "networks of networks" or multilevel networks. Recently, Wang et al. (2013) proposed an ERGM-based approach for studying such networks. In this model, several different classes of subgraph statistics are defined, given a two-level network of networks, which Wang et al. (2013) refer to as micro, macro, and meso networks.

In this framework, each class of networks consists of a set of structural properties considered within the model. One set corresponds to statistics involving individual networks defined at the "micro" level of analysis (for example, interpersonal relationships between and among team members), another set corresponds to networks at the "macro" level of analysis (for example, work product flows between teams), and a third set corresponds to the "meso" level - the bipartite affiliation network defining how individual nodes at one level are affiliated with nodes at the group level of analysis (for example, patterns of individual membership in different teams). Finally, more complex sets of statistics are defined based on structural patterns across these different networks.

This approach enables a number of new research questions involving networks. For example, researchers may be interested in the extent to which a node's popularity at one network level is associated with popularity in the second type of network (e.g., as indexed by affiliation-based popularity, Wang et al., 2013).

However, while the model is very general, and potentially quite powerful ${ }^{2}$, it may not necessarily be the best approach for all research questions involving multilevel networks. For instance, in some cases, research goals may be less concerned with modeling overall sets of micro-, macro-, and meso- level structure simultaneously, and more concerned with modeling variability in lower-level structures (e.g., cross-team variability in tendencies for reciprocity and centralization), or understanding relationships (possibly causal) between exogenous and endogenous networks at different levels of analysis. In other cases, the goal may be to allow the integration of ERGMs with the models and methods that are already dominant in particular fields. In such cases, a hierarchical Bayesian approach may prove very useful.

As a very simple example, we might be interested in testing hypotheses related to structural entrainment - modeling similarity, differences, and variance in micro-level structural features as a function of macro-level structure. For instance, given a particular multilevel network, we might wish to explore the extent to which groups that fill similar roles in the macro-level network (as defined by, e.g., structural equivalence in the macro-level network) have similar

\footnotetext{
${ }^{2}$ For example, even though the model is geared towards multilevel "networks of networks", it could also serve as a basis for the analysis of more general structures such as factor graphs with several types of modes. Multilevel networks might be usefully conceptualized as a special case of such graphs. An example of a multi-mode (but not necessarily multilevel) network includes Carley's person-task-knowledge meta-matrix (e.g., Carley, Pfeffer, Reminga, Storrick, \& Columbus, 2013).
} 
micro-level structural features. We might believe that micro-level networks in the "core" of the macro-level network will tend to have different structural features than those in the periphery of the macro-level network. Alternatively, we might expect that micro-level networks that are proximal to one another in the macro-level structure may be more likely to share certain types of structural tendencies, compared to groups that do not interact.

One straightforward way to approach these sorts of structural entrainment hypotheses might be to combine the RCERGM described above with a simple spatial autoregressive model (Leenders, 2002). In the usual spatial autoregressive (SAR) model, we have $\theta=\rho W \theta+X \beta+e$, where $W$ is the weight matrix (Doreian, 1982; Leenders, 2002) defining the nature of assumed dependence between observations (for example, based on spatial contiguity). The parameter $\rho$ is a regression weight that indexes the magnitude of the relationship given the data and assumed dependence structure. Following Haining (2003), and ignoring any prior distributions, this model can be written as follows:

$$
\begin{aligned}
g_{i} & \sim F\left(\theta_{i}, z_{i}\right) \\
\theta_{i} & \sim N\left((I-\rho W)^{-1} X \beta,(I-\rho W)^{-1} \sigma_{r}^{2}\left(I-\rho W^{\prime}\right)^{-1}\right)
\end{aligned}
$$

We can, if we wish, allow particular types of ERGM parameters (e.g., associated with edges, mutual dyads, etc.) to have their own sets of covariates and autocorrelation parameters.

\section{$3 \quad$ Example applications}

For purposes of exposition, we present sample analyses based on two data sets. The first data set, taken from (1 citation removed for masked review), is a sample of 14 networks where each network reflects the pattern of friendship relations among members of a particular person's social support network. These data are used to demonstrate the random coefficient ERGM and compare it to an analysis using a more traditional type of random-effects meta-analysis, based on separate group-wise ERGMs. The second analysis is based on simulated data; it is used to provide an example of the spatial entrainment model, and the integration of more complex higher-level dependencies among networks of networks.

All models below (except for the meta-analytic models) were estimated using the auxiliary variable MCMC method described by Liang (2010) and Caimo and Friel (2011). This baseline algorithm was augmented by the adaptive algorithm developed by Haario, Saksman, and Tamminen (2001). This algorithm is further described within Roberts and Rosenthal (2009), and studied within the context of social networks by Caimo and Mira (2015). The algorithm is discussed in more detail in the appendix. All meta-analytic models were fit using an adaptive Metropolis-Hastings algorithm (Haario et al., 2001). Details of estimation (run length, convergence) are discussed in each section below. 


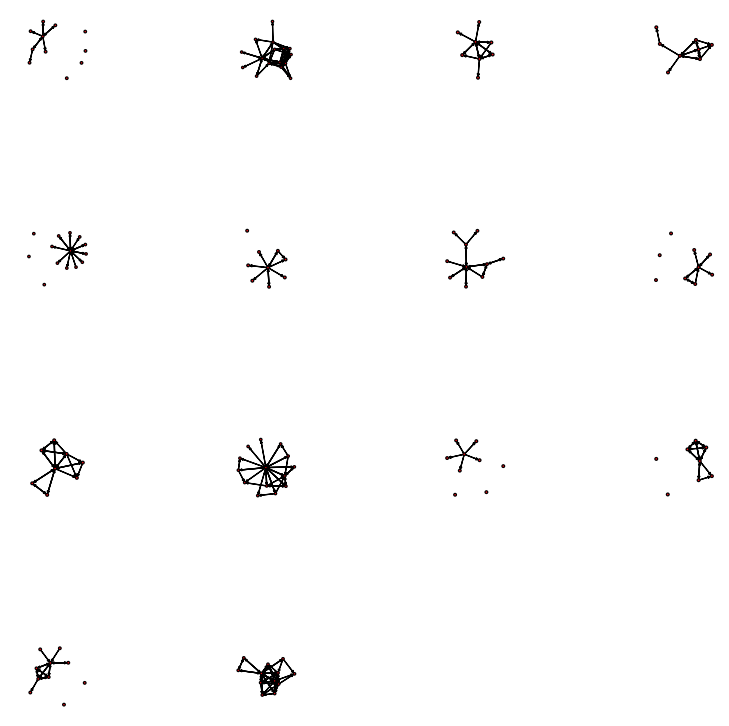

Figure 1: Friendship networks.

\subsection{Analyzing samples of networks: Friendship}

As an example of the basic random coefficient ERGM, we consider data taken from (1 citation removed for masked review): the original data set consisted of 29 friendship networks. Each network represents the friendship nominations involving a single cancer survivor and their social support network. To simplify estimation, networks with fewer than 8 people were dropped from the data set, leaving 14 networks. These networks are shown in Figure 1; they range in size from 8 people to 16 people, with a mean size of 10.4 and a standard deviation of 2.6.

To this set of networks, we fit four different models. All models included the same set of nine structural effects: edges, reciprocal dyads, in-stars of order two and three, out-stars of order two and three, mixed two-stars, transitive triads, and three-cycles. These are very complex models, fit to a small number of small networks.

Although extreme, this sort of setting is not uncommon in research on small groups such as teams and families, and presents a significant number of difficulties. First, very small networks may not have any observed graph statistics of a certain type (for example, no counts of reciprocal dyads). Second, the modeling of some of these graph statistics (e.g., counts of transitive triples) are known to be associated with a greater risk of degenerate models (Snijders, Pattison, Robins, \& Handcock, 2006). Of course, as with any statistical model with many 
parameters and small data sets, there is also a substantial risk of over-fitting ${ }^{3}$.

The first model (Model 1) represents a completely unpooled analysis. For our analyses, we take the basic ERGM described in Equations 1 and 2 and incorporate a vague prior for the ERGM parameters:

$$
\begin{aligned}
& g_{i} \sim F\left(\theta_{i}, z_{i}\right) \\
& P\left(\theta_{i}\right) \sim N(0,10000)
\end{aligned}
$$

The second model (Model 2) represents an application of a multivariate meta-analytic model (Jackson, Riley, \& White, 2011). We use the specification developed in Equation 3 and add vague priors for the mean and variance of the fixed effects and for the covariance matrix of random effects:

$$
\begin{aligned}
\theta_{i} & \sim M V N\left(\mu_{i}, \Sigma_{s e(i)}\right) \\
\mu_{i} & \sim M V N\left(\theta_{f}, \Sigma_{r}\right) \\
\theta_{f} & \sim N(0,10000) \\
\Sigma_{r} & \sim I W(9, I)
\end{aligned}
$$

In this model, $\theta_{i}$ is defined in terms of the mean of the posterior estimates of $\theta_{i}$ from Model 1. The covariance of the posterior estimates of the ERGM parameters for each graph $i$ are used to define $\Sigma_{s e(i)}$. All other model parameters are estimated freely.

Model 3 represents the full hierarchical Bayesian model without covariates. This model is described using the following set of equations:

$$
\begin{aligned}
g_{i} & \sim F\left(\theta_{i}, z_{i}\right) \\
\theta_{i} & \sim M V N\left(\theta_{f}, \Sigma_{r}\right) \\
\theta_{f} & \sim N(0,10000) \\
\Sigma_{r} & \sim I W(9, I)
\end{aligned}
$$

Finally, Model 4 uses group size (standardized) as a group-level predictor of the group-specific random effects. This model is defined as:

$$
\begin{aligned}
g_{i} & \sim F\left(\theta_{i}, z_{i}\right) \\
\theta_{i} & \sim M V N\left(\mu_{i}, \Sigma_{r}\right) \\
\mu_{i} & =X \beta \\
\beta_{f} & \sim N(0,10000) \\
\Sigma_{r} & \sim I W(9, I)
\end{aligned}
$$

Tests of the models using multiple chains found that the samplers generally converged very quickly, as defined by potential scale reduction factors close to

\footnotetext{
${ }^{3}$ This last issue falls outside of the scope of the current paper, but remains conceptually important for the larger issues of multi-model inference - e.g., comparing and selecting from among competing theoretical models.
} 
1.0 (Gelman \& Rubin, 1992); with a few exceptions, random and fixed effects generally converged within a few tens of thousands of iterations; elements of the random effects covariance matrix typically took much longer to reliably converge, requiring on the order of a million iterations or more. To ensure adequate burn-in for all parameters in all models, each model was estimated using three chains. Each chain was run for 30 million iterations, and thinned by a factor of 3000, resulting in 10000 recorded iterations for each chain. The last 5000 iterations of each chain were used for analysis.

To assess model fit, the simulate function (Handcock et al., 2003) of statnet was used. simulate was applied to the full posterior distribution of random effects, and the graph statistics for each newly simulated network were recorded. This provided a set of 15,000 simulated graph statistics for each of the 14 networks. Based on the graph statistics from the original networks and the simulated networks, various posterior predictive checks (c.f., Gelman, Meng, \& Stern, 1996; Meng, 1994) were then calculated.

To provide an example of shrinkage in the estimates, the random effects estimates associated with the reciprocity parameter for each of the 14 networks are presented in Table 1. The results show the expected pattern for an analysis of a complex model estimated from the small networks frequently encountered in research on small groups such as teams and families. First, the unpooled Bayesian estimates include a number of extreme parameter estimates with correspondingly large uncertainties for certain networks. Second, the table clearly shows how - as expected - all of the random-effects approaches strongly shrink those network-specific estimates towards a common mean, with more shrinkage for estimates with great uncertainty, and less shrinkage for estimates with less uncertainty.

These patterns highlight the fact that attempting to fit relatively complex models to such small networks independently of one another can prove very difficult. This is not surprising, since many of the observed networks do not contain particular sets of graph statistics. In a typical ML analysis (e.g., via statnet), many of those estimates would be fixed to some value (such as $-\infty$ ), rather than estimated; the Bayesian analysis produces estimates, but the use of a vague prior for the parameter estimates does not provide much regularization, and the estimates for many networks remain quite extreme (albeit with correspondingly large standard errors, representing appropriate levels of uncertainty).

An analysis of the estimated fixed effects from Models 2, 3, and 4 are presented in Table 2; the relationship between group size (standardized) and network-level random effects for Model 4 are also presented in Table 2. In many cases, fixed-effects estimates appeared roughly similar across the different models, with similar patterns of significance/non-significance.

However, as the table shows, there were some differences across models. For example, estimates for the in-star parameter of order 2 varied from 0.02 in Model 1 to -0.83 in Model 4 , and estimates for the out-star parameter of order 2 varied from 0.77 to 1.21. In Model 4, there appeared to be a significant relationship between group size and random-effect estimates of in-star parameters of order 


\begin{tabular}{rclll}
\hline & Model 1 & Model 2 & Model 3 & Model 4 \\
\hline 1 & $95.72(46.25)$ & $3.60(0.94)$ & $3.36(0.67)$ & $3.39(0.71)$ \\
2 & $2.04(1.75)$ & $3.48(0.74)$ & $3.08(0.66)$ & $2.36(0.94)$ \\
3 & $67.06(40.43)$ & $3.67(0.92)$ & $3.41(0.63)$ & $3.94(0.80)$ \\
4 & $1.09(1.88)$ & $3.42(0.77)$ & $3.23(0.65)$ & $3.65(0.79)$ \\
5 & $81.32(45.74)$ & $3.62(0.90)$ & $3.21(0.71)$ & $2.81(0.84)$ \\
6 & $62.28(42.06)$ & $3.57(0.87)$ & $3.30(0.68)$ & $3.71(0.79)$ \\
7 & $5.55(1.89)$ & $3.74(0.74)$ & $3.61(0.60)$ & $3.84(0.63)$ \\
8 & $83.37(53.88)$ & $3.63(0.91)$ & $3.52(0.63)$ & $3.83(0.70)$ \\
9 & $5.76(2.52)$ & $3.74(0.78)$ & $3.67(0.62)$ & $4.25(0.80)$ \\
10 & $3.31(1.36)$ & $3.58(0.72)$ & $3.48(0.60)$ & $3.09(0.73)$ \\
11 & $-14.23(109.15)$ & $3.65(0.92)$ & $3.14(0.74)$ & $3.49(0.85)$ \\
12 & $6.55(3.07)$ & $3.73(0.84)$ & $3.65(0.61)$ & $4.09(0.77)$ \\
13 & $7.64(3.00)$ & $3.70(0.79)$ & $3.71(0.62)$ & $3.84(0.68)$ \\
14 & $3.54(1.21)$ & $3.60(0.68)$ & $3.51(0.58)$ & $3.40(0.60)$ \\
\hline
\end{tabular}

Table 1: Estimated reciprocity parameters for all networks

2, with larger networks tending to have a larger estimate.

\begin{tabular}{rrrrr}
\hline Parameter & \multicolumn{1}{l}{ Model 2 } & Model 3 & \multicolumn{1}{l}{$\begin{array}{l}\text { Model 4 } \\
\text { Intercept }\end{array}$} & \multicolumn{1}{l}{$\begin{array}{l}\text { Model 4 } \\
\text { Group size }\end{array}$} \\
\hline Edge & $-3.57(0.61)$ & $-3.98(0.33)$ & $-3.96(0.38)$ & $-0.48(0.36)$ \\
Mutual & $3.62(0.64)$ & $3.42(0.42)$ & $3.55(0.46)$ & $-0.48(0.43)$ \\
In-star (2) & $0.02(0.40)$ & $-0.46(0.30)$ & $-0.83(0.37)$ & $0.59(0.30)$ \\
In-star (3) & $-0.04(0.21)$ & $-0.09(0.17)$ & $-0.01(0.20)$ & $-0.03(0.17)$ \\
Out-star (2) & $0.77(0.36)$ & $1.16(0.26)$ & $1.21(0.29)$ & $-0.08(0.26)$ \\
Out-star (3) & $-0.20(0.21)$ & $-0.36(0.14)$ & $-0.42(0.16)$ & $0.13(0.14)$ \\
Mixed 2-star & $-0.07(0.23)$ & $0.15(0.17)$ & $0.23(0.20)$ & $-0.07(0.18)$ \\
Transitive & $0.04(0.24)$ & $-0.11(0.19)$ & $-0.04(0.22)$ & $-0.24(0.18)$ \\
triples & & & & \\
3-cycles & $-0.31(0.47)$ & $-0.36(0.39)$ & $-0.49(0.48)$ & $-0.01(0.38)$ \\
\hline
\end{tabular}

Table 2: Fixed effects for Models 2, 3, and 4.

The estimated variances of all random effect parameters are shown in Table 3. Variances across the different models were generally similar. Point estimates of the random effects variances tended to be slightly larger for several of the ERGM coefficients, but these differences were not significant. Standard errors of the estimates tended to be smaller in the hierarchical Bayesian models (Models 3 and 4) than in the meta-analytic model.

An analysis of the correlations among random effects did not find significant differences among the three models. Examining the correlations among random effects, there were estimated minimum correlations of $-0.29,-0.40$, and -0.49 and estimated maximum correlations of $0.07,0.42$, and 0.39 for Models 2,3 , and 4 respectively. No correlations were significant, which is not surprising given the 
small number of groups in the model.

\begin{tabular}{rlll}
\hline Parameter & Model 2 & Model 3 & Model 4 \\
\hline Edge & $0.41(0.33)$ & $0.44(0.28)$ & $0.52(0.28)$ \\
Mutual & $0.47(0.53)$ & $0.36(0.19)$ & $0.39(0.24)$ \\
In-star (2) & $0.39(0.37)$ & $0.42(0.22)$ & $0.45(0.23)$ \\
In-star (3) & $0.25(0.21)$ & $0.17(0.08)$ & $0.17(0.07)$ \\
Out-star (2) & $0.41(0.40)$ & $0.40(0.21)$ & $0.45(0.21)$ \\
Out-star (3) & $0.25(0.19)$ & $0.16(0.06)$ & $0.19(0.08)$ \\
Mixed 2-star & $0.25(0.18)$ & $0.21(0.09)$ & $0.24(0.11)$ \\
Transitive & $0.31(0.22)$ & $0.25(0.11)$ & $0.24(0.10)$ \\
triples & & & \\
3-cycles & $0.41(0.35)$ & $0.30(0.15)$ & $0.31(0.15)$ \\
\hline
\end{tabular}

Table 3: Variance components for Models 2, 3, and 4.

Model fit was assessed by examining posterior predicted network statistics. First, each of the four models was examined with respect to how well networks generated from the full posterior distribution of the random effects replicated the observed model statistics (counts of edges, reciprocal dyads, etc.). Despite the complicated model and extremely small networks, the models appeared to provide a generally acceptable fit for most networks, at least in terms of reproducing the original statistics.

In fact, out of the 126 total graph statistics (14 graphs x 9 network statistics) being estimated for each model, the observed network statistics fell outside of the $80 \%$ credibility intervals of the posterior predictive replications only a few times for each of the four models (four times for Model 1, six times for Model 2, twice for Model 3, and five times for Model 4). These problematic estimates were almost entirely associated with network 2 .

A closer examination of network 2 found that this network appeared to display much greater numbers of certain kinds of dyadic and triadic configurations than any of the other 14 networks: specifically, asymmetric and null dyads, in-2stars, out-2-stars, and transitive triads. In an applied setting, this type of model fit information could be used to help guide the development of new models by identifying specific aspects of the observed network that are not reliably reproduced by the specific statistical model under consideration. For example, the misfit of network 2 to the larger multilevel model may indicate that we should rethink the assumption that network 2 comes from the same distribution as the rest of the networks. This use of posterior predictive checks is consistent with current practices for assessing model fit in Bayesian models (Gelman et al., 2013).

Figure 2 shows the distribution of posterior predictive p-values (PPP-values) across all networks and statistics for each of the four models. PPP-values are defined by the proportion of times simulated values for a particular graph statistic exceeded the observed value for particular networks. The mean PPP-values across all parameters were $0.45,0.62,0.48$, and 0.49 for Models 1-4, respec- 


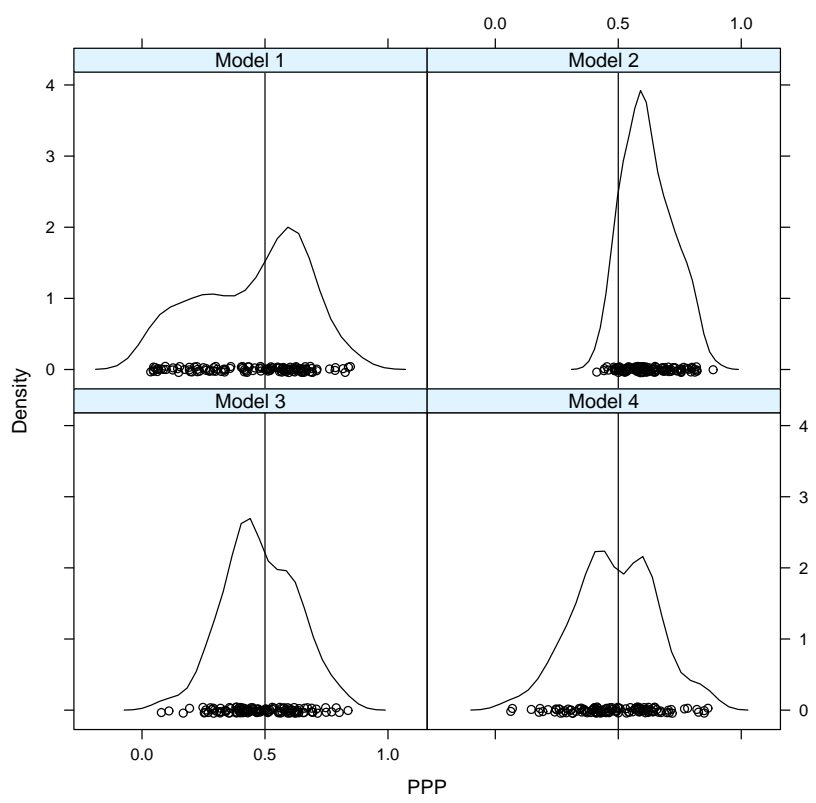

Figure 2: Distribution of posterior predictive p-values for all graph statistics across all networks, Models 1-4. Each PPP-value indicates the proportion of times a simulated network statistic exceeded the observed statistic. The vertical line at 0.5 indicates the expected PPP value when the simulated graph statistics of the posterior predictive networks are correctly centered on the graph statistics of the observed networks.

tively. PPP-values for all statistics for all models fell within 0.025 and 0.975 . For Models 2-4, most PPP-values fell within 0.2 to 0.8. For Model 1, several $\mathrm{PPP}$-values were less than 0.2 ; these were largely associated with graphs where certain statistics were not observed (for example, network 11, where no reciprocal dyads were observed). For such cases, the bulk of the simulated values were also zero, leading to relatively small PPP-values.

The mean square error (MSE) was calculated separately for each of the nine statistics, comparing the distribution of posterior predicted network statistics to the observed statistics for each of the 14 networks. For Models 1-4, the total MSE values across all networks and parameters were $9.5 \times 10^{6}, 24.7 \times 10^{6}$, $8.9 \times 10^{6}$, and $6.8 \times 10^{6}$, respectively. Thus, using total MSE on the modeled graph statistics as a criterion, the two hierarchical Bayesian models appeared to provide the best overall fit, followed by the unpooled model, and the metaanalytic model.

Interestingly, the random-effects meta-analytic model produced MSE estimates an order of magnitude worse than the other three models. Given that most random and fixed effects appeared roughly similar across the various mod- 
els, and that Model 2 appeared to provide generally good fit in terms of the credibility intervals of posterior predictive statistics and the PPP-values, this large difference in MSE was surprising. An examination of the posterior predictive simulations identified the source of this extreme misfit: a tendency for Model 2 (the meta-analytic model) to produce degenerate networks when simulating from the full posterior of the model, for almost every network.

To illustrate, Figure 3 shows a plot of the density of the simulated graphs for each of the 4 models, based on the random effects associated with a sample of networks (networks 1, 2, and 7). Plots showing the distribution of posterior predictive graph statistics for all models and all networks can be found in the supplementary materials. An examination of these plots suggests some clear patterns.

First, all models (Models 1-4) appeared to have difficulty fitting network 2, at least in terms of reproducing the overall density. This confirms the earlier model fit results. Second, while no model was completely free of degeneracy in this very difficult sample, Model 2 produced highly bimodal distributions of posterior graph statistics for almost every network in the sample, and had a tendency to produce simulated networks with densities at or near 1.0 for almost every single network in the data set, despite having parameter estimates for the fixed and random effects that appeared reasonable. Third, Model 1 appeared to have a slight tendency to overproduce empty graphs (networks with density at or near 0.0) compared to the other models.

Overall, our results indicate that - at least in this particular data set and model - the use of a hierarchical modeling approach provided the best ability to replicate the observed graph statistics, and the smallest tendency to produce degenerate graphs from the posterior distribution of the model parameters. Estimates from the meta-analytic model were generally very close in magnitude to estimates from the fully hierarchical model, but with a few small differences. More importantly, full posterior parameter estimates from the meta-analytic model had a distinct tendency to produce degenerate networks.

\subsection{Analyzing multilevel networks: Network-level struc- tural entrainment}

To provide an example of an application of the structural entrainment model described earlier, as well as to highlight an application of the current models to a less difficult data set, we produced a simulated data set involving a network of networks. We began by simulating 30 sets of networks, along with a single upper-level network. The structural entrainment model defines a network of networks where we allow for the possibility that the structural features of a lower-level network may depend on the structural features of other networks with which it shares a connection in a higher-level network.

The upper-level network, shown in Figure 4 was drawn from a Bernoulli random graph distribution with tie probability of 0.05 . This network was rowstandardized; this standardization defined the weight matrix $W$. To generate the lower-level networks, we simulated values $\theta_{j}=\mu_{j}+\rho_{j} W \theta_{j}+e_{j}$ for $j=1,2,3$. 


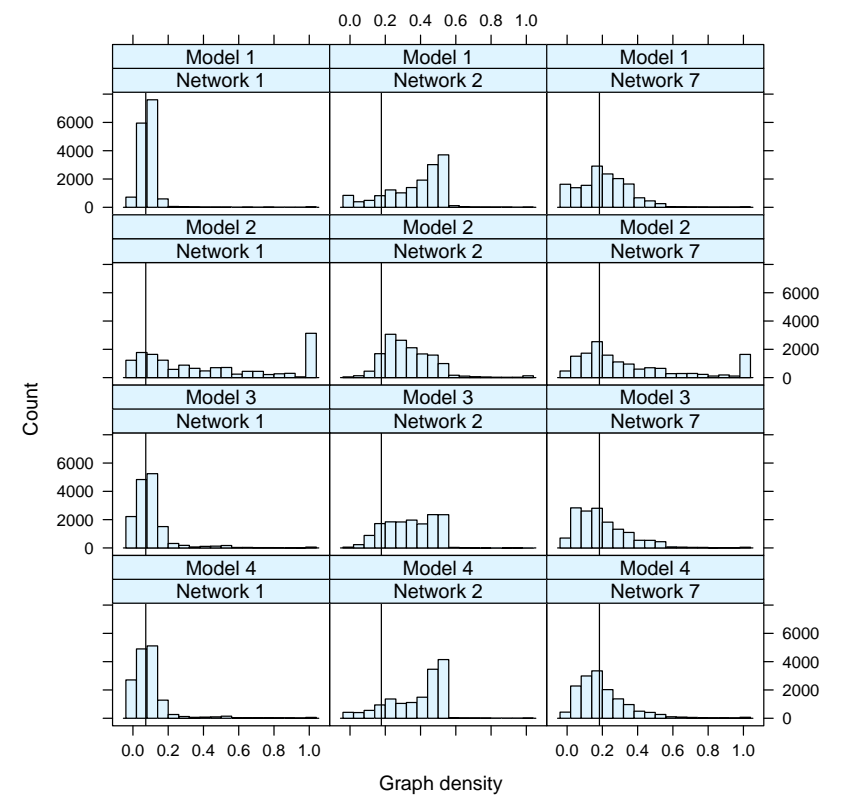

Figure 3: Histogram of the posterior predictive network density, for networks 1, 2 , and 7 . The vertical line indicates the density of the observed networks. 


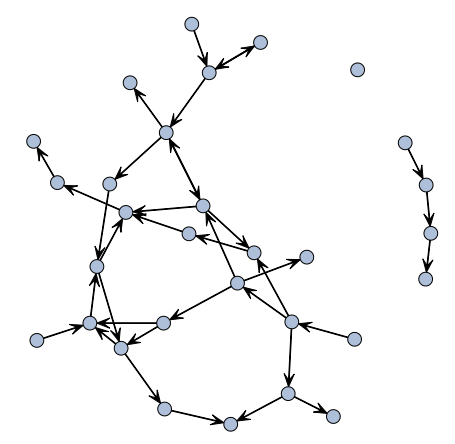

Figure 4: Upper-level network, used to define dependency structures between simulated lower-level networks.

The values of the fixed effects, $\mu_{j}$ were $-1.0,0.8$, and -0.3 for edges, reciprocal dyads, and transitive triads, respectively. The autocorrelation $\rho_{j}$ associated with each of these three parameters was set to $0.4,0.0$, and 0.0 , which provided a mix of values with and without autocorrelation. The standard deviation of the random effects $\theta_{j}$ was set at $0.3,0.4$, and 0.2 .

These values were used to generate 30 lower-level networks, using the simulate function of statnet. Each lower-level network contained 20 nodes. An example of one of these simulated lower-level networks is shown in Figure 5. Copies of the generated networks (both upper- and lower-level) are provided in the supplementary materials, along with the ERGM parameter values used to simulate them.

These simulated networks were then analyzed using four different models. Models 1-3 of this section are roughly analogous to Models 1-3 from the previous section, with three exceptions. First, random effects $\theta_{i}$ were treated as uncorrelated and normally distributed, to match the generating mechanism just described: $\theta_{i} \sim N\left(\theta_{f}, \sigma_{r}^{2}\right)$. Second, the variance components $\sigma_{r}^{2}$ were given a large uniform prior, $\sigma_{r}^{2} \sim U(0,10000)$. Third, the underlying ERGM incorporated only three parameters instead of nine.

Model 4 represents the structural entrainment model, designed to allow for network autocorrelation among random effects associated with lower-level ERGM estimates. We model each of the $j=1,2,3$ ERGM parameters for each of the $i=1 . . .30$ networks using the standard ERGM. We model the mean and variability of ERGM parameters across networks using the SAR specification (Haining, 2003), giving us the following statistical model: 


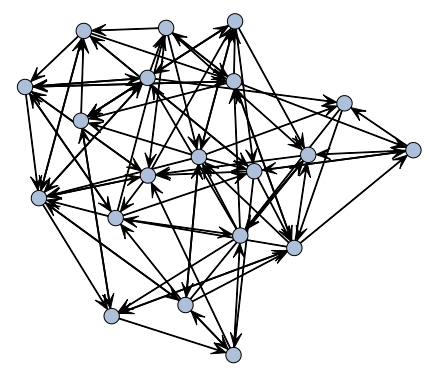

Figure 5: Example of lower-level network.

$$
\begin{aligned}
& g_{i} \sim F\left(\theta_{i}, z_{i}\right) \\
& \theta_{i} \sim N\left((I-\rho W)^{-1} \mu,(I-\rho W)^{-1} \sigma_{r}^{2}\left(I-\rho W^{\prime}\right)^{-1}\right) \\
& P(\mu) \sim N(0,10000) \\
& P(\rho) \sim U(-1,1) \\
& P\left(\sigma_{\text {random }}^{2}\right) \sim U(0,10000)
\end{aligned}
$$

In this model, $W$ is the row-normalized weight matrix defined using the upper-level network (Figure 4); $\rho$ represents the vector of autocorrelation parameters. Since the weight matrix is row-standardized, this is constrained to the interval $|\rho|<1$ (Haining, 2003). Other parameters are defined as in previous models: $\sigma_{\text {random }}^{2}$ is a vector of three variance parameters (one for each of the three model parameters) and $\mu$ represents a vector of intercept terms which define the fixed effects.

The same algorithms from the previous section were used to estimate these models. Models 1,3 , and 4 tended to converge very quickly - usually within a few thousand iterations for the random effects. The variance and autocorrelation parameters took slightly longer. Model 2 took substantially longer to converge - several tens of thousands of iterations for most parameters, with much higher autocorrelations in the chains.

Each model was estimated using three chains. Chains for each of Models 1, 3 , and 4 were run for a total of a million iterations, thinned by a factor of 100 , for a total of 10000 iterations per chain. Due to slower convergence, Model 2 was run for a total of 30 million iterations, thinned by a factor of 3000 , for a total of 10000 iterations per chain. The last 5000 iterations of each of the three chains was used for purposes of estimation. Posterior predictive graph statistics 
were generated in the same way as the previous section for all networks and all models.

In terms of model fit, most models reproduced the observed graph statistics reasonably well for most networks. For Models 2-4, an examination of network statistics produced by simulating new networks from the posterior distribution of the random effects found no evidence of excessive numbers of complete or null graphs. Model 1, the completely unpooled solution, displayed a minor tendency to produce complete graphs from the posterior simulations for certain networks.

The posterior predictive p-values across each of the 30 graphs and 3 network statistics are shown in Figure 6. PPP values appeared to be acceptable for most statistics from most networks, with means of $0.42,0.45,0.45$, and 0.45 for Models 1-4. The MSE was calculated for different targets, which are shown in Table 4. Table 4 shows that the hierarchical Bayesian model and the structural entrainment model (Models 3 and 4) were the best in terms of fitting the graph statistics of the observed networks.

\begin{tabular}{rllll}
\hline MSE target & Model 1 & Model 2 & Model 3 & Model 4 \\
\hline Graph statistics & $79.1 \times 10^{5}$ & $17.2 \times 10^{5}$ & $2.5 \times 10^{5}$ & $2.4 \times 10^{5}$ \\
Fixed effects & & 0.30 & 0.31 & 0.11 \\
SD & & 0.09 & 0.09 & 0.06 \\
\hline
\end{tabular}

Table 4: MSE estimated for various aspects of the original data. MSE for the fixed effects and variance components (SD) are computed using the values used to generate the underlying networks.

Table 5 shows a sample of the random effects estimates for the edge parameter across the four different models, along with the original values that were used to help generate the simulated networks. Compared to Models 1-3, estimates from the autoregressive model (Model 4) tended to be slightly closer to the original values on average, and have slightly smaller standard errors. This is reflected by the values in Table 4, where the MSE for the random effects was lowest for the structural entrainment model (Model 4).

The fixed effects, variance components, and autocorrelation parameters are shown in Table 6 , along with the values used to simulate the underlying network data. Estimates for the fixed effects estimates and variance components were generally very similar for Models 2 and 3, which is reflected in the nearly identical MSE for those components (Table 4).

However, MSE values also indicate that the spatial entrainment model provided much better estimates of the fixed effects, which can be seen in Table 6 . Specifically, the models that did not allow for autocorrelation across networks tended to produce estimates of the edge parameter that were significantly lower than the originating value, and with smaller standard errors. The structural entrainment model produced estimates close to the original value for the edge parameter, and for the autocorrelation parameter.

The difference in estimates between Model 4 and Models 1-3 demonstrates the potential danger of failing to explicitly model dependencies between lower- 


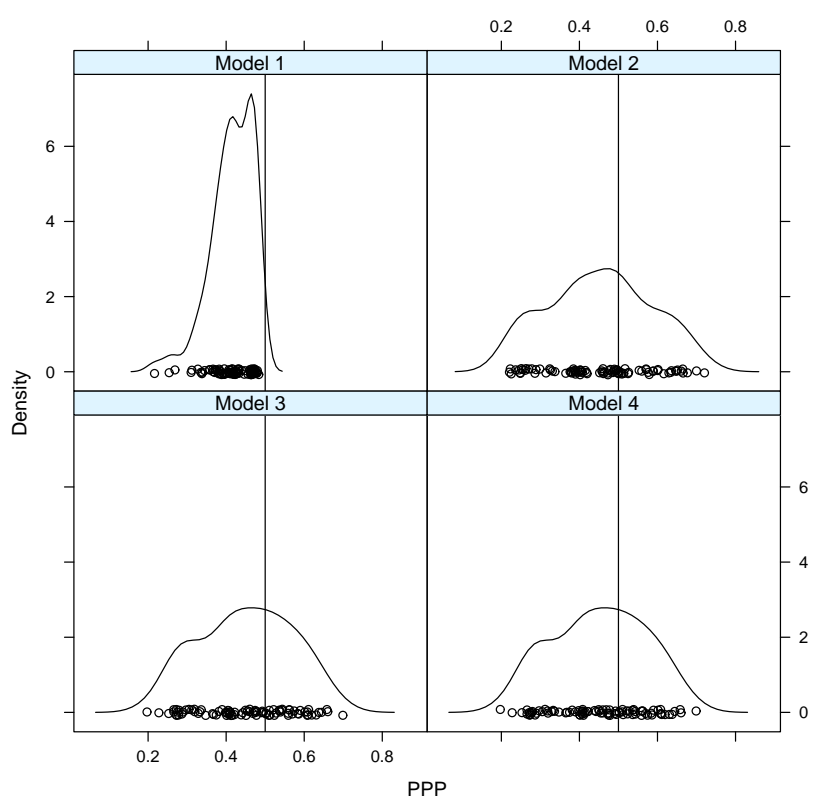

Figure 6: Distribution of posterior predictive p-values across all 30 networks and 3 network statistics. The vertical line indicates the target value of 0.5 . 


\begin{tabular}{rlllll}
\hline Network & $\begin{array}{l}\text { Original } \\
\text { value }\end{array}$ & Model 1 & Model 2 & Model 3 & Model 4 \\
\hline 1 & -0.91 & $-0.31(0.33)$ & $-0.66(0.26)$ & $-0.68(0.26)$ & $-0.78(0.25)$ \\
2 & -1.51 & $-0.88(0.32)$ & $-1.22(0.24)$ & $-1.22(0.23)$ & $-1.28(0.21)$ \\
3 & -1.72 & $-1.56(0.27)$ & $-1.45(0.22)$ & $-1.44(0.23)$ & $-1.44(0.22)$ \\
4 & -1.35 & $-1.33(0.30)$ & $-1.48(0.22)$ & $-1.49(0.22)$ & $-1.51(0.20)$ \\
5 & -1.61 & $-2.19(0.25)$ & $-1.94(0.20)$ & $-1.93(0.20)$ & $-1.93(0.20)$ \\
6 & -1.01 & $-0.73(0.32)$ & $-1.22(0.24)$ & $-1.23(0.23)$ & $-1.26(0.22)$ \\
7 & -0.99 & $-0.91(0.33)$ & $-1.12(0.24)$ & $-1.12(0.23)$ & $-1.03(0.23)$ \\
8 & -1.41 & $-1.68(0.28)$ & $-1.67(0.21)$ & $-1.66(0.21)$ & $-1.72(0.20)$ \\
9 & -0.75 & $-0.33(0.32)$ & $-0.80(0.25)$ & $-0.82(0.25)$ & $-0.74(0.24)$ \\
10 & -1.53 & $-2.09(0.25)$ & $-1.90(0.19)$ & $-1.92(0.20)$ & $-1.89(0.19)$ \\
\hline
\end{tabular}

Table 5: Edge parameter estimates for a sample of simulated networks.

level networks when such dependence exists; in this case, the presence of autocorrelation leads to biased fixed-effects estimates for models that did not incorporate that aspect of statistical dependence.

\begin{tabular}{rrrrrr}
\hline & Parameter & $\begin{array}{l}\text { Original } \\
\text { Value }\end{array}$ & \multicolumn{1}{c}{ Model 2 } & \multicolumn{1}{c}{ Model 3 } & \multicolumn{1}{c}{ Model 4 } \\
\hline Fixed & Edge & -1.00 & $-1.51(0.11)$ & $-1.52(0.11)$ & $-1.14(0.20)$ \\
& Reciprocity & 0.80 & $0.87(0.13)$ & $0.87(0.13)$ & $0.72(0.19)$ \\
& Transitive & -0.30 & $-0.31(0.05)$ & $-0.31(0.05)$ & $-0.34(0.07)$ \\
triples & & & & \\
\hline SD & Edge & 0.30 & $0.53(0.09)$ & $0.53(0.09)$ & $0.48(0.09)$ \\
& Reciprocity & 0.40 & $0.50(0.13)$ & $0.51(0.13)$ & $0.48(0.12)$ \\
& Transitive & 0.20 & $0.18(0.05)$ & $0.18(0.04)$ & $0.17(0.05)$ \\
& triples & & & & \\
\hline$\rho$ & Edge & 0.30 & & & $0.34(0.15)$ \\
& Reciprocity & 0.00 & & & $-0.24(0.23)$ \\
& Transitive & 0.00 & & & \\
triples & & & &
\end{tabular}

Table 6: Estimates of fixed effects, standard deviations of random effects, and autocorrelation parameters.

Compared to the results from the first example using the real-world friendship networks, estimates based on the simulated networks were less problematic overall. This is not suprising, since they involved much larger networks (20 nodes per network), a much larger set of networks (30), much fewer parameters (3), and first-stage estimates of the network-level parameters that were generally very well-behaved.

In this situation, the meta-analytic model (Model 2) worked nearly as well as Model 3 (the fully Bayesian hierarchical model), at least in terms of recovering the original parameters used to generate the model; model fit in terms of 
recovering the observed graph statistics was still slightly worse. Finally, Model 1 (the unpooled model) displayed a small tendency for posterior estimates to occasionally produce simulated networks that were complete (estimated densities at or near 1.0). No such tendency was observed in Models 2-4.

\section{Discussion}

The application of Bayesian techniques to study social networks is certainly not new. In fact, a wide variety of models have been developed that use hierarchical Bayesian techniques to make inferences about various aspects of graph structure. Examples of this have included applications to cognitive social structures (Butts, 2003), exponential random graph models for single networks (Jin et al., 2013; Schweinberger \& Handcock, 2013), and p2 models for samples of networks (Zijlstra et al., 2006).

When studying the structure of a single network, the use of Bayesian techniques may provide some benefits in terms of parameter estimation Caimo and Friel (2011); however, of potentially greater importance is that such approaches may allow for increased flexibility in specifying and testing more complex models. This includes multilevel models designed to account for structural variation and dependencies in both independent samples of networks and networks-ofnetworks with complex patterns of dependency.

The Bayesian models proposed here for samples of networks, although very simple, could have important applications; this includes applications to the study of the structure of small groups (e.g., teams, classrooms, and families) and its relationship to group-level factors (e.g., contextual covariates like type of team task, or experimental interventions designed to change group behavior). In this context, reliably applying traditional single-network models to large numbers of small networks can be challenging, and estimates based on post-hoc meta-analytic approaches may - in some circumstances - be potentially unreliable.

By contrast, a fully Bayesian approach to modeling sets of networks allows for random effects estimates that flexibly incorporate prior information about the possible distribution of ERGM coefficients across networks, provides both estimates of random and fixed effects that take into account the full posterior uncertainty across all model parameters, and affords for an elegant way to impute missing data in both actor covariates and network ties. Models within a hierarchical Bayesian context can be easily modified and expanded as needed to incorporate new types of covariates and more complicated models (see Draper, 1995).

This flexibility could provide a number of uses. The ability to more easily incorporate network-level variables may make the study of network structure in experimental or quasi-experimental contexts more feasible. This can be accomplished, for example, via the straightforward application of a regressiondiscontinuity approach within the random effects ERGM model. Other applications might include the development of new Bayesian mixture models. 
Such models could extend current network mixture approaches geared towards the clustering of actors according to latent proximity (Handcock, Raftery, \& Tantrum, 2007) or structural equivalence (Salter-Townshend \& Murphy, 2015) by allowing for clustering of network parameters across multiple types of relationships and networks. Such models would be potentially useful for identifying latent classes of groups defined by similarity in social structure.

There are no conceptual barriers to developing even more complex hybrid models. For example, random effects extensions to the basic Wang et al. (2013) multilevel model can potentially be developed to account for differences and variability in graph statistics at the micro-network or meso-network level of analysis. Additionally, models can be adapted to allow for additional flexibility in modeling uncertainty in networks, where such uncertainty might be related to measurement error in node covariates, or possibly even uncertainty in the size of the network itself.

Of course, there are important caveats to the proposed models. One of the most important is the assumption of exchangeability across the set of networks. Future work will need to identify the conditions under which exchangeability is an appropriate assumption, and how that is best translated into modeling terms.

In certain cases, such as when groups are all of equal sizes, and there are no expectations of dramatic differences in the scale of network statistics across groups of different size, the basic assumptions used here might be reasonable. In other situations, appropriate choices of covariates or changes to distributional assumptions (for example, to allow for random effects that follow a $t$ - or skew- $t$ distribution) might be necessary. While the proposed models are flexible enough to allow for varying distributional specifications, research is needed on how such distributional assumptions - including prior specifications - impact the efficiency and accuracy of parameter estimates.

In addition, the auxiliary variable MCMC routines used to estimate these models can be computationally expensive. Moreover, multilevel models can frequently induce additional challenges, such as poor mixing for certain parameters. Finding ways to improve efficiency in the sampler can be difficult.

Finally, and most importantly, much more work needs to be done on assessing model fit. It is not always straightforward to assess fit in either ERGM or multilevel contexts. Determining the most appropriate ways to assess fit for models such as the random coefficient or structural entrainment ERGMs is even more challenging.

In many respects, the models presented in this paper are very basic. However, in the case of small group research, they may also be quite valuable providing a modeling framework for studying variability across networks. In particular, hypotheses regarding the role of network-level covariates on structural patterning within networks can be easily specified and tested. This could lead to a better understanding of how social structures vary within and across groups. 


\section{Appendix}

The sorts of models described above are easily implemented using Bayesian approaches to exponential random graph models (e.g., Caimo \& Friel, 2011; Jin et al., 2013), such as certain auxiliary variable MCMC methods. In the current paper, we make use of the auxiliary variable sampler described in Liang (2010) and Caimo and Friel (2011), with additional modifications to improve sampler performance.

This approach is comprised of a few basic steps:

1. Begin MCMC sampler with initial state $\theta_{t}$

2. Propose new values $\theta_{t+1}$ for model parameters, given some proposal distribution - for example, a multivariate normal distribution with some covariance $\Sigma$, centered on the current value $\theta_{t}$

3. Given proposed values $\theta_{t+1}$, generate an auxiliary network $G_{a u x}$ from the original network $G$.

4. Accept proposed $\theta_{t+1}$ with probability $\alpha$, or reject and retain previous values $\theta_{t}$, where $\alpha=\frac{P\left(G \mid \theta_{t+1}\right)}{P(G \mid \theta t)} \times \frac{P\left(G_{a u x} \mid \theta_{t}\right)}{P\left(G_{a u x} \mid \theta_{t+1}\right)} \times \frac{P\left(\theta_{t+1}\right)}{P\left(\theta_{t}\right)}$.

\section{Repeat $2-4$}

This basic algorithm works very well for a single network, given an appropriate choice of proposal distributions. In practice, a good choice might include $2.4 / d^{2} * \mathrm{R}$, where $\mathrm{R}$ is the estimated covariance among parameters, taken from the maximum likelihood or maximum pseudolikelihood estimates, and $\mathrm{d}$ is the number of parameters being estimated in the ERGM. For generating the auxiliary network, we use a series of metropolis steps, as described in Snijders (2002). We have experimented with the number of steps used to generate the auxiliary network; in many applications, a single round of metropolis steps (e.g., k steps for a network with k edges or arcs) often appears to provide good performance, although the convergence of the algorithm may not be guaranteed with such short steps (Jin et al., 2013).

The choice of how many steps to use is an area of current research. In our own experience, experimentation on several real-world data sets indicates that correlations between the observed network (the starting point of the auxiliary network generation routine) and the resulting auxiliary (simulated) network decreased quickly as the number of steps increased. In many cases, the correlations appear to asymptote to a minimum after a relatively small number of steps (3-5 iterations over each network). The analyses in this paper iterated over all ties in each network only 5 times, but experiments with larger numbers of iterations (e.g., 10, 100, and 1000) on the current data and models did not produce appreciably different results for parameter estimates.

The random coefficient ERGM and the multilevel network models presented in this paper contain a much larger number of parameters than a single model. In addition, certain aspects of multilevel models can sometimes bring additional 
problems with mixing and sampler performance. To improve performance, we make use of two additional strategies: a blockwise sampling strategy, and an adaptive MCMC strategy.

We separate the parameters into $b$ sets of blocks, including separate blocks for the random effects associated with each of the different networks. There is also a separate block of parameters for the fixed effects in the model (including network covariates), and a final block of parameters for the variance components associated with the random effects. The algorithm proceeds by proposing (then accepting/rejecting) parameters block-by-block.

We begin the algorithm using pre-specified distributions to generate the proposals; in the case of the models used here, we use a multivariate normal proposal distribution for all blocks of parameters. The initial proposal distribution for parameters in each block uses a pre-specified covariance matrix, with diagonal elements set to some small value (e.g., 0.0001), and off-diagonal elements set to zero.

After the sampler has performed some set number of iterations, the proposal distribution changes to the adaptive proposal of Haario, Saksman, and Tamminen (2001). This involves keeping a running update of the covariance matrix of the MCMC chain, and using that to provide the covariance matrix for future proposals. Each block is tracked and updated separately.

\section{References}

Anderson, C. J., Wasserman, S., \& Crouch, B. (1999). A p* primer: Logit models for social networks. Social Networks, 21(1), 37-66.

Butts, C. T. (2003). Network inference, error, and informant (in)accuracy: A bayesian approach. social networks, 25(2), 103-140.

Caimo, A., \& Friel, N. (2011). Bayesian inference for exponential random graph models. Social Networks, 33(1), 41-55.

Caimo, A., \& Mira, A. (2015). Efficient computational strategies for doubly intractable problems with applications to bayesian social networks. Statistics and Computing, 25(1), 113-125.

Carley, K., Pfeffer, J., Reminga, J., Storrick, J., \& Columbus, D. (2013). Ora user's guide 2013 [Computer software manual].

Debray, T. P., Moons, K. G., Abo-Zaid, G. M. A., Koffijberg, H., \& Riley, R. D. (2013). Individual participant data meta-analysis for a binary outcome: One-stage or two-stage? PloS one, 8(4), e60650.

Desmarais, B. A., \& Cranmer, S. J. (2012). Statistical mechanics of networks: Estimation and uncertainty. Physica A: Statistical Mechanics and its Applications, 391 (4), 1865-1876.

Doreian, P. (1982). Maximum likelihood methods for linear models spatial effect and spatial disturbance terms. Sociological Methods 6 Research, 10(3), 243-269.

Draper, D. (1995). Assessment and propagation of model uncertainty. Journal of the Royal Statistical Society. Series B (Methodological), 45-97. 
Dutton, M. (2011). Individual patient-level data meta-analysis: A comparison of methods for the diverse populations collaboration data set (Unpublished doctoral dissertation). Florida State University.

Gelman, A., Carlin, J., Stern, H., Dunson, D., Vehtari, A., \& Rubin, D. (2013). Bayesian data analysis (3rd ed.). CRC Press.

Gelman, A., Jakulin, A., Pittau, M. G., \& Su, Y.-S. (2008). A weakly informative default prior distribution for logistic and other regression models. The Annals of Applied Statistics, 1360-1383.

Gelman, A., Meng, X.-L., \& Stern, H. (1996). Posterior predictive assessment of model fitness via realized discrepancies. Statistica Sinica, 6(4), 733-760.

Gelman, A., \& Rubin, D. B. (1992). Inference from iterative simulation using multiple sequences. Statistical science, 457-472.

Haario, H., Saksman, E., \& Tamminen, J. (2001). An adaptive metropolis algorithm. Bernoulli, 223-242.

Haining, R. P. (2003). Spatial data analysis: Theory and practice. Cambridge University Press.

Handcock, M. S., Hunter, D. R., Butts, C. T., Goodreau, S. M., \& Morris, M. (2003). statnet: Software tools for the statistical modeling of network data. Retrieved from http://statnetproject.org

Handcock, M. S., Raftery, A. E., \& Tantrum, J. M. (2007). Model-based clustering for social networks. Journal of the Royal Statistical Society: Series A (Statistics in Society), 170(2), 301-354.

Heinze, G., \& Schemper, M. (2002). A solution to the problem of separation in logistic regression. Statistics in medicine, 21(16), 2409-2419.

Hunter, D. R., Krivitsky, P. N., \& Schweinberger, M. (2012). Computational statistical methods for social network models. Journal of Computational and Graphical Statistics, 21(4), 856-882.

Jackson, D., Riley, R., \& White, I. R. (2011). Multivariate meta-analysis: Potential and promise. Statistics in Medicine, 30(20), 2481-2498.

Jin, I. H., Yuan, Y., \& Liang, F. (2013). Bayesian analysis for exponential random graph models using the adaptive exchange sampler. Statistics and its interface, 6(4), 559-576.

Koehly, L. M., \& Pattison, P. (2005). Random graph models for social networks: Multiple relations or multiple raters. Models and methods in social network analysis, 162-191.

Leenders, R. T. A. (2002). Modeling social influence through network autocorrelation: Constructing the weight matrix. Social Networks, 24(1), 21-47.

Liang, F. (2010). A double metropolis-hastings sampler for spatial models with intractable normalizing constants. Journal of Statistical Computation and Simulation, 80(9), 1007-1022.

Lubbers, M. J., \& Snijders, T. A. (2007). A comparison of various approaches to the exponential random graph model: A reanalysis of 102 student networks in school classes. Social Networks, 29(4), 489-507.

Lunn, D. J., Thomas, A., Best, N., \& Spiegelhalter, D. (2000). Winbugs a bayesian modelling framework: Concepts, structure, and extensibility. Statistics and computing, 10(4), 325-337. 
Lusher, D., Koskinen, J., \& Robins, G. (Eds.). (2013). Exponential random graph models for social networks: Theory, methods, and applications. Cambridge University Press.

Marin, J.-M., Pudlo, P., Robert, C. P., \& Ryder, R. J. (2012). Approximate bayesian computational methods. Statistics and Computing, 22(6), 11671180.

Meng, X.-L. (1994). Posterior predictive p-values. The Annals of Statistics, $1142-1160$.

Morris, C. N., \& Lysy, M. (2012). Shrinkage estimation in multilevel normal models. Statistical Science, 27(1), 115-134.

Roberts, G. O., \& Rosenthal, J. S. (2009). Examples of adaptive mcmc. Journal of Computational and Graphical Statistics, 18(2), 349-367.

Salter-Townshend, M., \& Murphy, T. B. (2015). Role analysis in networks using mixtures of exponential random graph models. Journal of Computational and Graphical Statistics, $24(2), 520-538$.

Schweinberger, M., \& Handcock, M. S. (2013). hergm, an $r$ package suggested by the statnet suite. Retrieved from http://cran.r-project.org/web/packages/hergm/

Snijders, T. A. (2002). Markov chain monte carlo estimation of exponential random graph models. Journal of Social Structure, 3(2), 1-40.

Snijders, T. A., Pattison, P. E., Robins, G. L., \& Handcock, M. S. (2006). New specifications for exponential random graph models. Sociological methodology, 36(1), 99-153.

Stan Development Team. (2013). Stan: A $c++$ library for probability and sampling, version 2.1.

Steyerberg, E., Eijkemans, M., \& Habbema, J. (2001). Application of shrinkage techniques in logistic regression analysis: A case study. Statistica Neerlandica, 55(1), 76-88.

Wang, P., Robins, G., Pattison, P., \& Lazega, E. (2013). Exponential random graph models for multilevel networks. Social Networks.

Wang, P., Sharpe, K., Robins, G. L., \& Pattison, P. E. (2009). Exponential random graph $\left(\mathrm{p}^{*}\right)$ models for affiliation networks. Social Networks, 31(1), $12-25$.

Zijlstra, B. J., van Duijn, M. A., \& Snijders, T. A. (2006). The multilevel p2 model. Methodology: European Journal of Research Methods for the Behavioral and Social Sciences, 2(1), 42-47. 